\title{
Role of Resistant Starches Particularly Rice Containing Resistant Starches in Type 2 Diabetes
}

\author{
Analava Mitra*, D. Bhattacharya** and S. Roy* \\ * B.C. Roy Technology Hospital, **Chemical Engineering Department, Indian Institute of \\ Technology, Kharagpur 721302, West Bengal, India \\ E-mail: amitra@adm.iitkgp.ernet.in
}

KEYWORDS Diabetes. Rice Protein. Starch. Cholesterol

\begin{abstract}
Non-insulin-dependent or type 2 diabetes mellitus is on the rise in India particularly in the affluent urban and rural population. The exact cause is unknown. Malnutrition, defective intake of essential fatty acids and reduced intake of fibres are found in Indian diet, particularly rural Indian diet and the dietary factors may be one of the causes leading to increased incidence of the disease. Dietary fibers reduce blood glucose and cholesterol levels. Resistant starches behave as dietary fiber. As rice is the staple food in Indian villages, to increase the fibre consumption, a special variety of rice is processed which contains more of resistant starch. The high protein efficiency ratio (PER) in rice is presumably due to the balance of essential amino acids in rice protein. This high resistant starch containing rice was fed to diabetic patients for three months and effects observed. Results showed that total cholesterol, fasting blood sugar, low-density lipoprotein cholesterol were reduced while other parameters showed no significant changes.
\end{abstract}

\section{INTRODUCTION}

Food starches may be either glycaemic or resistant. Glycaemic starches are those that are degraded to glucose by enzymes in the digestive tract and can be further sub-classified as rapidly digestible starch (RDS) or slowly digestible starch (SDS). The example of RDS is mashed potatoes, which is hydrolyzed to glucose within $20 \mathrm{~min}$. An example of SDS is raw starch from wheat and is degraded more slowly than RDS. The period for conversion of SDS into glucose may vary from 20 to $100 \mathrm{~min}$. Resistant starches (RS) are those that are not digested in the small intestine and are fermented in the large intestine by bacterial microflora define Phillips et al. (1995).

Resistant starches show potential health benefits and are similar to fiber and show promising physiological benefits in humans, which may result in much disease prevention and reduction of sufferings. Fiber has been shown to have health benefits in controlling or preventing carcinomas and heart disease. It causes reduction of blood glucose level in

Address for correspondence: Analava Mitra, B-145 IIT Campus, Indian Institute of Technology, Kharagpur 721302, West Bengal, India

Telephone: 091-0322-279970

Fax: 091-03222-282631

E-mail: amitra@adm.iitkgp.ernet.in diabetes as observed by Gordon et al. (1997). Its functional properties such as improved crispness and expansion in certain products and better mouth feel, color, and flavor have drawn broad interest worldwide.

Englyst et al. (1992) classified RS into three groups, which are RS1, RS2, and RS3 on the basis of factors intrinsic to the food source or by processing treatment of the food. RS1 consists of enclosed starch such as partially milled grains; seeds and legumes and the seed or outer coating must be broken for digestion process. Examples of RS2 are starches found in green bananas, uncooked potatoes etc. RS2 is un-gelatinized and cannot be digested by amylases till it is gelatinized. RS3 is present in bread, cooked potatoes, and ready-to-eat breakfast cereals and includes nongranular starches that have either been retrograded or crystalline in structure.

Resistant starches behave as total dietary fiber (TDF) as refer Johnson and Gee (1996) and are measured by the approved AOAC method for fiber analysis. Although it is not a fiber, resistant starch can be defined as a "functional fiber," as analyzed for nutritional labeling. The current dietary recommendation is of 20-30 g of fiber per day as mention Yue and Waring (1998). Present day intake varies between 12 and $17 \mathrm{~g}$ because $\phi$ fiber-fortified foods' lack appeal, as fibers are having a strong flavor and are being unpalatable. Further, they possess a coarse texture and dry mouth feel. Nutritional Labeling 
and Education Act (NLEA) classifies fibers as per serving (serving sizes vary depending on the food) and calls it a "good source" if it contains $2.5 \mathrm{~g}$ of fiber per serving and a "high source" if it contains $5.0 \mathrm{~g}$ of fiber per serving. European Community calls it more if it has more than $2 \%$ total dietary fiber (TDF), an "increased" fiber content if it contains more than 3\%, and "high or rich" in fiber if it has more than 6\% TDF observe Yue and Waring (1998).

Ali and Bhattacharya (1976 b and 1980) concluded that a part of gelatinized starch seems to retrograde during slow drying of parboiled rough rice. Depending on retro-gradation component, parboiled rice is more hydrated and solubilized than raw rice at low temperature but less so at higher temperatures. Hydration power of rice is unusually high immediately after steaming and gradually falls to normal value during air-drying observe Ali and Bhattacharya (1976), Priestley (1976 and 1977). Parboiled rice exhibits greater slurry viscosity at room temperature and is in conformity of greater hydration at low temperature observe Unnikrishnan and Bhattacharya (1983); Ali and Bhattacharya (1976a). It was observed that when parboiled rough rice after steaming was immediately dispersed by homogenization in water, the apparent solubility of rice substance increased with increasing severity of steaming, but the values assumed a reverse order and the highest solubility was in raw rice when the parboiled samples were dried, milled and recooked. This shows that a certain extent of reassociation occurs in rice starch soon after the steaming step in the parboiling process. The extent of re-association depends on both the moisture content and the storage temperature (Ali and Bhattacharya, 1976b). At usual, uniform moisture retro-gradation is of about $25 \%$ at room temperature, about $30 \%$ at $8{ }^{\circ} \mathrm{C}$ and $20 \%$ at $50^{\circ} \mathrm{C}$. The paradox may be related to the intermediate moisture content of parboiled rice. At high moisture content, alignment of starch molecules prior to any association is unstable due to rapid molecular vibration and so retro-gradation is retarded by heating and hastened by cooling. At low moisture contents, a suitable alignment of the sluggish molecules is helped by increased molecular vibration caused by heating and hence retro gradation is favored by heating.

Diabetes mellitus, its complications and diseases associated with it are common globally.
The most common variety, i.e., non-insulin dependent diabetes mellitus (NIDDM), is again a disease of plenty and approximately 30 million people or 2-5\% adult populations are its victims. The trend is on the rise (King, 1998). NIDDM is commonly associated with hypertension, dyslipidaemia, accelerated atherosclerosis and increased incidence of coronary artery disease. Essential Hypertension, common due to stressful patterns of life, is most common in western life. $25 \%$ of adult populations are the sufferers. As per 1997 statistics, 691 million people worldwide have hypertension as suggests Capra (1983), 135 million NIDDM; the later is estimated to increase to 300 million by 2025 says King (1997). Indians have a genetic predisposition to accelerated atherosclerosis. This is further enhanced by change in lifestyle or 'westernisation', according to Enase and other speakers at a plenary symposium of the annual conference of the Cardiological Society of India on 'High Rate of Coronary Artery Disease in Indians: A Global Perspective' at Calcutta in November (1997). The combination of "nature" (genetics) and "nurture" (environment) could explain the high and early occurrence of coronary artery diseases among Indians. While body mass index in Indians was not higher, they had a significantly higher waisthip ratio finds Gupta et al (1996) indicating that although Indians have no more general obesity than the others they tend to put it on centrally or abdominally. They also had more hyperinsulinaemia, glucose intolerance and plasminogen activator inhibitor 1 (PAI-1). Regarding the antioxidants, Indians had a lower level of plasma vitamin $\mathrm{C}$ and selenium. These factors could be due to food habits, especially prolonged cooking at higher temperatures, and cooking with ù- 6 oils as the main oil medium with a myth to reduce cholesterol level as suggests Raheja (1999). Average Indian diabetic and CAD patients were younger. Diabetes mellitus, insulin resistance, low HDL, and high triglycerides in Indians replace smoking, hypertension and high cholesterol in westerners at risk.

A traditional villager in India does not have access to a good physician, well-equipped hospital, good diagnostic centers, and proper educational back up in management of chronic diseases. Neutraceuticals, a global idea, are food substances or ingredients that confer medical or health benefits. Tomorrow's consumer will seek to 
include health-promoting ingredients in their diets, antioxidants and vitamins, fibres and mucilage, minerals like calcium, zinc, chromium, selenium and iron, flavonoids and phytochemicals, fish oil or its equivalents, vegetable proteins of good quality, herbal products such as spices etc. protecting their all important right to eat and enjoy.

\section{MATERIAL AND METHODS}

The following materials were used for the study- Raw rice (Jaya variety of Boro rice after 30 minutes of milling), Common Balance (Addy and Co. in Kolkata), Earthenware containers from local market, Gunny bag from local market, Equipments for parboiled rice obtained locally, manual and, photometer 4010 from Boehringer Manheim, reagents and tourniquets from BCRT Hospital in Kharagpur.

Retrograded rice was prepared as per traditional practices followed in rural Bengal. $40 \mathrm{~kg}$ of Rice, is subjected to heating as in case of conventional method of parboiling but cooling was done very slowly by covering it in a gunny bag and keeping it in a specially designed containers made up of earthenware about 6 feet in length, 1 feet in breadth and 9 inches in height where rice is filled upto 6 inches, for about 12 hours till it attains a brownish hue. The rice was being inspected continuously throughout the procedure, so that the desired texture was not lost. Rice thus produced was tested for qualitative and quantitative analysis of retrograded starch in Central Research Facility (CRF) by x-ray diffraction, at I.I.T. Kharagpur. The main disadvantage was that the prepared rice was reddish in colour and required longer time to cook and cost of cooking energy was high. The storage quality was also poor.

40 randomly selected newly detected type 2 diabetic patients were screened and they were placed in 2 homogenous groups of 20 patients each. To one group (group 1), the samples thus prepared was tested and the other group (group 2 ) acts as control. The patients with known renal and hepatic dysfunction and on lipid lowering therapy were excluded from the study. The patients underwent clinical, anthropometrical evaluation before the study. Written consent of patients were taken before the study, A dietary survey of the patents was done by asking questionnaire and it was found that on an average the patients eat $150 \mathrm{~g}$ of rice per day. Instead of usual rice they consume, the patient's in-group 1 was given to eat $150 \mathrm{~g}$ of the rice thus prepared per day. The other group took usual and normal food including same variety of rice purchased from market and not processed as described. Both the groups maintained identical life styles and activities during the period of study. Blood samples were taken at monthly intervals by an indwelling catheter into ante-cubital vein and analyzed for fasting blood glucose (FBS), triglyceride (TG), total cholesterol (TLC), highdensity lipoprotein cholesterol (HDLC), lowdensity lipoprotein cholesterol (LDLC), very low-density lipoprotein cholesterol (VLDLC) by standard methods as depicted by Boehringer Mannheim (1983) and photometer 4010. The study continued for three months and effects in the blood parameters as mentioned were observed after taking blood samples of all the volunteers at monthly intervals.

\section{RESULTS AND DISCUSSION}

The patients underwent clinical, anthropometrical evaluation before the study. The observations were:

Sex: Males-28, Females-12,

Age: $45.32 \pm 4.25$ years,

Weight: $69.46 \pm 7.32 \mathrm{~kg}$,

Body Mass Index (BMI): $22.32 \pm 5.23$.

At the end of study clinical, anthropometrical evaluations were also done. The results showed negligible variations except body weight and BMI which showed variation of $\pm 0.33 \mathrm{~kg}$ and \pm 0.23 respectively and this may be attributed to non-identical conditions pertaining at the time of taking measurements.

Raw rice samples were analysed at Food Analysis Laboratory of Agriculture and Food Engineering Department of I.I.T.-Kharagpur. It was found that $100 \mathrm{~g}$ of rice, prepared by the process contains $6 \mathrm{~g}$ of protein, $1 \mathrm{~g}$ of fat, $45 \mathrm{~g}$ of carbohydrate in addition to calcium, iron and other minerals based on the methods described on Rangana (1986). Retrograded starch content in the samples was 8-10\% (Central Research Facility, IIT, Kharagpur).

The results are presented in Table 1, which showed that TLC values were reduced from 230 $\mathrm{mg} / \mathrm{dl}$ to $210 \mathrm{mg} / \mathrm{dl}(\mathrm{p}=<0.025)$, FBS values were reduced from $158 \mathrm{mg} / \mathrm{dl}$ to $140 \mathrm{mg} / \mathrm{dl}(\mathrm{p}=<0.050)$ and LDLC values were reduced from $164 \mathrm{mg} / \mathrm{dl}$ to $144 \mathrm{mg} / \mathrm{dl}(\mathrm{p}=<0.025)$ respectively, while other 
parameters showed no significant changes. Table 2 showed that with normal diet, different parameters of lipid profile such as, HDLC, LDLC, VLDLC and TG remained unaffected while TLC and LDLC showed an increase of about $6 \mathrm{mg} / \mathrm{dl}$ which may be attributed to rural diet (Mitra and Bhattacharya, 2005). FBS showed practically no changes. The observations are pertinent with village life style patterns. Analysis of fasting serum insulin values in the group receiving more of resistant starches through rice was $94 \pm 26$ units (initially) and it was $102 \pm 18$ units at the end of study and so it may be concluded the effects in fasting blood sugar may be due to increased serum insulin level, needing further studies. Reduction in TLC and LDLC may be due to increase fibre effects (Macrae et al., 1993). Younes et al. (1995) reported that resistant starch reduces cholesterol without significant reduction of HDLC and triglycerides.

Rice protein has a high protein efficiency ratio (PER) and is presumably due to the balance of essential amino acids in rice protein. Also, rice proteins derived from brown or partially polished rice contain bran, which can lower cholesterol as already observed by Seetharamaiah and Chandrashekhara (1990). Many of these benefits are related to a specific physiological response to a particular rice component, for example the oryzanols in rice oil as have been found to be by Seetharamaiah and Chandrashekhara (1990). Extensive research and studies with both animal and human subjects have clearly demonstrated rice bran and rice bran oil's hypocholesterolemic effect as remark Kahlon et al. (1996); Kahlon and Chow (1997) and Nicolosi et al. (1991). Specific rice-bran fractions showing this effect include rice-bran oil and the unsaponifiable fraction. The unsaponifiable fraction, both the tocotrienols and the oryzanols, has also demonstrated strong antioxidant activity. Rice-bran fiber is also expected to have the well-known benefits of fiber. In addition, rice bran hemicellulose has been shown to have unique biological activity. The soluble fraction from rice bran has been shown to assist in controlling blood sugar levels.

\section{CONCLUSIONS}

The study clearly demonstrates that a little modification of rice processing can alter the characteristics of rice. This rice fills the bulk, that is, the satiety but majority being nonabsorbable does not cause rise in blood sugar value. Further due to the fiber and other effects as discussed, total and LDL cholesterol was reduced. The process is not costly and does not involve much expertise. The only problem is slight but acceptable change in taste and increase fuel cost to cook the rice.

\section{ACKNOWLEDGEMENT}

The authors are indebted to Late Prof. S. K. Sawarkar of Department of Chemical Engineering, Indian Institute of Technology, Kharagpur, India for his valuable suggestions and encouragement throughout the work. The authors are also deeply indebted to Prof. H. N. Mishra and Prof. S. K. Das of Agriculture and Food Engineering Department of Indian Institute of Technology, Kharagpur, India for his review, comments and suggestions.

Table 1: Effects of consuming rice containing resistant starch

\begin{tabular}{lllllll}
\hline $\begin{array}{l}\text { Time } \\
(\text { month })\end{array}$ & $\begin{array}{c}T L C \\
(m g / d l)\end{array}$ & $\begin{array}{c}H D L C \\
(m g / d l)\end{array}$ & $\begin{array}{c}L D L C \\
(m g / d l)\end{array}$ & $\begin{array}{l}V L D L C \\
(m g / d l)\end{array}$ & $\begin{array}{c}T G \\
(m g / d l)\end{array}$ & $\begin{array}{c}F B S \\
(m g / d l)\end{array}$ \\
\hline 0 & $230 \pm 17$ & $40 \pm 9$ & $164 \pm 17$ & $26 \pm 9$ & $130 \pm 21$ & $158 \pm 12$ \\
1 & $222 \pm 12$ & $40 \pm 11$ & $156 \pm 12$ & $26 \pm 7$ & $130 \pm 17$ & $152 \pm 17$ \\
2 & $216 \pm 14$ & $42 \pm 7$ & $148 \pm 15$ & $26 \pm 7$ & $130 \pm 17$ & $144 \pm 13$ \\
3 & $210 \pm 16$ & $42 \pm 7$ & $144 \pm 17$ & $24 \pm 5$ & $120 \pm 17$ & $140 \pm 15$ \\
\hline
\end{tabular}

Table 2: The values of 20 NIDDM patients who were on normal diet and not receiving resistant starch.

\begin{tabular}{lcccccc}
\hline $\begin{array}{l}\text { Time } \\
(\text { months })\end{array}$ & $\begin{array}{c}T L C \\
(m g / d l)\end{array}$ & $\begin{array}{c}H D L C \\
(m g / d l)\end{array}$ & $\begin{array}{c}L D L C \\
(m g / d l)\end{array}$ & $\begin{array}{l}\text { VLDLC } \\
(m g / d l)\end{array}$ & $\begin{array}{c}T G \\
(m g / d l)\end{array}$ & $\begin{array}{c}F B S \\
(m g / d l)\end{array}$ \\
\hline 0 & $180 \pm 32$ & $48 \pm 12$ & $104 \pm 26$ & $28 \pm 19$ & $136 \pm 21$ & $152 \pm 20$ \\
1 & $180 \pm 38$ & $48 \pm 12$ & $104 \pm 17$ & $28 \pm 14$ & $140 \pm 24$ & $152 \pm 17$ \\
2 & $186 \pm 24$ & $48 \pm 19$ & $112 \pm 21$ & $26 \pm 13$ & $130 \pm 17$ & $150 \pm 21$ \\
3 & $185 \pm 32$ & $48 \pm 17$ & $110 \pm 31$ & $27 \pm 11$ & $135 \pm 21$ & $154 \pm 12$ \\
\hline
\end{tabular}




\section{REFERENCES}

Ali, S. and Bhattacharya, K.R.: Comparative; properties of ration rice and parboiled rice. Hebenson Wiss Technol., 9: 11-13 (1976a).

Ali, S. and Bhattacharya. K.R.: Starch retrogradation and starch damage in parboiled rice and flaked rice. Staerke, 28: 233-240 (1976b).

Ali, S. and Bhattacharya, K.R.: Changes in sugars and amino acids during parboiling of rice. J. Food Biochem., 4: 169-179 (1980).

Boehringer Mannheim. Instruction sheets for manual assays. GmbH diagnostics: Catalogue no.124095 (cholesterol), diagnostics Catalogue no 543004 (HDLC), diagnostics Catalogue no 124966 (Triglycerides), Catalogue no.263 826 (Glucose), Catalogue no.400 971 and catalogue no. 543004 (LDLC and VLDLC). Germany: 6800 Mannheim 31, Boehringer Mannheim GmbH 1983.

Capra, Fritjof: The Turning Point. Flamingo, London (1983)

Enase, Ae: High Rate of Coronary Artery Disease in Indians: A Global Perspective. Keynote address. Annual Conference of the Cardiological Society of India, Calcutta (1997).

Englyst, H. N., Kingman. S. M. and Cummings. J. H.: Classification and measurement of nutritionally important starch fractions. Euro. J. Clin. Nutr., 46 (Suppl. 2): S 33 (1992).

Gordon, D.T., Topp, K., Shi, Y.-C., Zallie, J. and Jeffcoat, R: Resistant starch: physical and physiological properties. Pp. 10-13. In: New Technologies for Healthy Foods and Nutraceuticals. M. Yalpani (Eds). ATL Press, Inc. Science Publishers, Shrewsbury, MA, New York (1997)

Gupta, R., Prakash, H. and Majumdar, S.: Prevalence of Coronary Heart Disease and Coronary Risk Factors in an Urban Population of Rajasthan. Indian Heart Journal, 47: 331-338 (1996).

Johnson, I. T. and Gee, J. M.: Resistant starch. Nutr. Food Sci., 1: 20 (1996).

King Hilary: Prevalence of Diabetes will increase 195\% in India by the year 2025. Presidential Address. IX National Congress of Diabetes. Mumbai (1998).

Kahlon, T. S., Chow, F. I., Chiu. M. M. and Hudson. C. A.: Cholesterol-lowering in hamsters by rice bran and rice bran oil unsaponifiable matter. Cereal Chem., 73: 69 (1996).

Kahlon, T. S and Chow F. I.: Hypocholesterolemic effects of oat, rice and barley dietary fibers and fractions. Cereal Foods World, 42: 86 (1997).

Macrae, R., Robinson, R.K., Sadler, M.J.: Encyclopedia of Food Science, Food Technology and Nutrition, $2^{\text {nd }}$ volume: $925-945$ and 1362-1387. Academic Press, London 1993.

Mitra, A and Bhattacharya, D: Effects of overall consumption, dietary patterns,cooking, on patients suffering from Non insulin dependent diabetes mellitus. J. of Interacad., 9(4): 635-642 (2005).

Nicolosi, R. J., Ausman, L. M and Hegsted, D. M.: Rice bran oil lowers serum total and low ensity lipoprotein cholesterol and apo B levels in nonhuman primates. Atherosclerosis, 88: 133 (1991).

Priestley, R.J.: Studies on parboiled rice, Comparison of the characteristics of raw and parboiled rice. Food Chem., 1: 5-14 (1976).

Priestley, R. J.: Studies on parboiled rice, characteristics of parboiled rice on recooking. Food Chem., 2: 4350 (1977).

Rangana, S.: Hand Book of Analysis and Quality Control for the Fruit and Vegetable Products. Tata McGraw Hills Limited, New Delhi (1986).

Seetharamaiah, G.S and Chandrasekhara, N.: Effect of oryzanol on cholesterol absorption and biliary and fecal bile acids in rats. Indian J. Med. Res., 92: 471 (1990).

Unnikrishnan, K. R and Bhattacharya, K.R.: Cold-slurry viscosity of processed rice flour. J. Texture Stnd., 14: 21-30 (1983)

Younes, H., Levrat, M-A, De'migne, C. and Re'mse'y, C.: Resistant starch is than Cholestyramine as a Lipid-Lowering agent in the Rat. Lipids, 30(9): 847853 (1995)

Yue, P and Waring, S.: Resistant Starch in Food Applications. Cereal Food World, 692(43): 9 (1998). 